\title{
Polyvinylpyrrolidone - Reduced Graphene Oxide - Pd Nanoparticles as An Efficient Nanocomposite for Catalysis Applications in Cross-Coupling Reactions
}

\author{
Hany A. Elazab ${ }^{1,2, *}$, Tamer T. El-Idreesy ${ }^{3,4}$ \\ ${ }^{1}$ Department of Chemical Engineering, Faculty of Engineering, The British University in Egypt, \\ El-Shorouk City, Cairo, Egypt
}

${ }^{2}$ Nanotechnology Research Centre (NTRC), the British University in Egypt (BUE), El-Sherouk City, Suez Desert Road, Cairo, 11837, Egypt

${ }^{3}$ Department of Chemistry, Faculty of Science, Cairo University, Giza 12613, Egypt

${ }^{4}$ Department of Chemistry, School of Sciences and Engineering, The American University in Cairo, New Cairo 11835, Egypt

Received: 23rd October 2018; Revised: 14 th March 2019; Accepted: $20^{\text {th }}$ March 2019;

Available online: 30th September 2019; Published regularly: December 2019

\begin{abstract}
This paper reported a scientific approach adopting microwave-assisted synthesis as a synthetic route for preparing highly active palladium nanoparticles stabilized by polyvinylpyrrolidone (Pd/PVP) and supported on reduced Graphene oxide (rGO) as a highly active catalyst used for Suzuki, Heck, and Sonogashira cross coupling reactions with remarkable turnover number (6500) and turnover frequency of $78000 \mathrm{~h}^{-1}$. Pd/PVP nanoparticles supported on reduced Graphene oxide nanosheets (Pd-PVP/rGO) showed an outstanding performance through high catalytic activity towards cross coupling reactions. A simple, reproducible, and reliable method was used to prepare this efficient catalyst using microwave irradiation synthetic conditions. The synthesis approach requires simultaneous reduction of palladium and in the presence of Gaphene oxide (GO) nanosheets using ethylene glycol as a solvent and also as a strong reducing agent. The highly active and recyclable catalyst has so many advantages including the use of mild reaction conditions, short reaction times in an environmentally benign solvent system. Moreover, the prepared catalyst could be recycled for up to five times with nearly the same high catalytic activity. Furthermore, the high catalytic activity and recyclability of the prepared catalyst are due to the strong catalyst-support interaction. The defect sites in the reduced Graphene oxide (rGO) act as nucleation centers that enable anchoring of both $\mathrm{Pd} / \mathrm{PVP}$ nanoparticles and hence, minimize the possibility of agglomeration which leads to a severe decrease in the catalytic activity. Copyright (C) 2019 BCREC Group. All rights reserved
\end{abstract}

Keywords: Graphene; Cross-Coupling; Microwave-assisted synthesis; Heterogeneous catalysis; Catalyst recycling

How to Cite: Elazab, H.A., El-Idreesy, T.T. (2019). Polyvinylpyrrolidone - Reduced Graphene Oxide - Pd Nanoparticles as an Efficient Nanocomposite for Catalysis Applications in Cross-Coupling Reactions. Bulletin of Chemical Reaction Engineering \& Catalysis, 14(3): 490-501 (doi:10.9767/bcrec.14.3.3461.490-501)

Permalink/DOI: https://doi.org/10.9767/bcrec.14.3.3461.490-501

\section{Introduction}

Over the past few decades, Nanoscience has emerged as a new promising interdisciplinary scientific field [1-4]. Nanoclusters have been investigated for many catalytic applications due to their large surface-to-volume ratio. Recently,

* Corresponding Author.

E-mail: elazabha@vcu.edu (H.A. Elazab); one of the main interests that attracted researchers' attention is transition metals based materials, especially when using palladium nanoparticles [5-10]. It is well established that palladium-based catalysts have been widely used in homogeneous and heterogeneous catalysis due to their several outstanding properties that combine between those of single metal atoms and other bulk metals [11-16]. In order to design new compounds with tailored chemical and 
physical properties, it is of vital importance to control both the catalyst particle size as well as the catalyst particle size distribution [17-23]. The designed nanostructured materials were used as heterogeneous catalysts and their performance was compared to the traditional powder catalysts. Using those newly designed nanostructured materials led to extraordinary special properties with remarkable enhancement in activity due to controlling size, shape, reaction active sites, catalytic selectivity, and surface structure [24-26].

The unique properties of polymers if used in combination with other transition metals like palladium are considered as a decisive factor that cause palladium nanoparticles to be used as an ideal candidate for enormous applications in biomedical, chemical, physical, environmental, biological, and other engineering fields [2731]. Particularly, it was reported that polyvinylpyrrolidone (PVP) which is a cheap linear polymer could be used an efficient stabilizer that could easily stabilize palladium(0) nanoparticles. The high catalytic activity for metallic and bimetallic nanoparticles supported on Graphene nanosheets and its defect sites was recently reported by many researchers in the catalysis research field [32-37]. It is widely known that one of the most important processes in the field of organic synthesis is the carbon-carbon bond formation through crosscoupling reactions that are catalyzed by transition metals. Those types of reactions are widely used in many areas like agricultural, medicinal, and pharmaceutical applications [1113,23,38-42]. The new synthetic trends are mainly focused recently on using catalysis at the nano scale for green chemistry using microwave assisted synthesis [11-13,15,16,18,20,22, 23,40-46].

In this context, Pd-PVP has been reported to be one of the most efficient catalysts in cross coupling reactions. Recently, carbon supported catalysts based on the single atom thick layer of carbon atoms in two dimensional (2D) honeycomb lattice named as Graphene have been considered as an ideal support due to its unique thermal, mechanical, electronic structures, and also high surface area [15,16,39,47-53]. Furthermore, the outstanding characteristics of Gaphene enabled its combination with other metallic and bi-metallic nanoparticle catalysts which can be used in various chemical reactions and transformations. Moreover, the defects in the graphene lattice structure enabled an easy and excellent process of anchoring the metals like palladium at the nanoscale to the surface of Gaphene leading to tunable metal- support interaction through surface functionalities [11-16,50-52]. The role of polyvinylpyrrolidone (PVP) as a non-toxic, water soluble, and low-cost linear polymer is that it can simply enable forming film stability besides enhancing the chemical properties. The advantage of using PVP in combination with graphene is that PVP could interact with it by strong $\pi-\pi$ stacking interaction. The addition of PVP adds a protective layer to the palladium particles which inhibits any possibility of metal-metal particle bond formation. Hence, prevention of the palladium particles sintering or agglomeration is achieved [2-4,34].

There are several synthetic routes that were used in order to prepare graphene based PdPVP catalysts including but not limited to chemical, hydrothermal, microwave-assisted synthesis methods [54-58]. However, it is important to notice that it was reported in several research findings that the adopted method of preparation play a pivotal role in their catalytic activity. Also, most of reported data from literature is mainly focused on the application in Suzuki cross-coupling; however, we report here a comprehensive evaluation of the catalytic activity of the prepared catalysts for several potential applications in Suzuki, Heck, and Sonogashira cross-coupling [2-4].

This research was inspired by another synthetic route that was reported recently [2-4]. The aim of this work is to introduce a complete scientific study including the use of a new enhanced approach adopting microwave-assisted synthesis as a synthetic route for preparing highly active palladium nanoparticles stabilized by polyvinylpyrrolidone (Pd/PVP) and supported on reduced Graphene oxide (rGO) as a highly active catalyst used for Suzuki, Heck, and Sonogashira cross coupling reactions. Our adopted method is a kind of improvement for our recent work using microwave assisted synthesis to gain some favorable advantages including not only mild reaction conditions, short reaction duration, but also ligand free environmentally benign solvent system that provides high economic viability [11-16,50-52,68].

\section{Materials and Methods}

Chemicals were used as received without any other purification. High-purity graphite powder (99.9999\%, 200 mesh) was purchased from Alfa Aesar. Palladium nitrate (10 wt.\% in 10 wt.\% $\mathrm{HNO}_{3}, 99.999 \%$ ), ethylene glycol, aryl bromide, bromobenzene, and potassium carbonate were obtained from Sigma Aldrich. 


\subsection{Catalyst Preparation}

\subsubsection{Synthesis of Graphene Oxide (GO)}

Hummers and Offeman method was used to prepare Graphene Oxide (GO) [59-62]. In the adopted synthetic approach, the high-purity graphite powder was oxidized using a mixture of $\mathrm{H}_{2} \mathrm{SO}_{4} / \mathrm{KMnO}_{4}$. Graphite powder (4.5 g, 0.375 mol) and $\mathrm{NaNO}_{3}(2.5 \mathrm{~g}, 0.0294 \mathrm{~mol})$ were mixed together in a conical flask and then the entire mixture was kept in an ice bath under continuous stirring. Then, a solution of concentrated $\mathrm{H}_{2} \mathrm{SO}_{4}(115 \mathrm{~mL}, 2.157 \mathrm{~mol})$ followed by $\mathrm{KMnO}_{4}$ (15 g, $0.095 \mathrm{~mol}$ ) were slowly added over a period of $2.5 \mathrm{~h}$ followed by addition of deionized water $(230 \mathrm{~mL}$ while keeping the temperature of the mixture around $80{ }^{\circ} \mathrm{C}$. Then, the entire mixture was stirred for nearly $20 \mathrm{~min}$; and deionized water $(700 \mathrm{~mL})$ along with $(10 \%) \mathrm{H}_{2} \mathrm{O}_{2}$ (20 mL, $0.667 \mathrm{~mol})$ were added and then, the reaction mixture was stirred for another $5 \mathrm{~min}$. The resulting yellow-brownish cake was washed several times with $1 \mathrm{M} \mathrm{HCl}(20 \mathrm{~mL})$ followed by deionized water $(3 \mathrm{~L})$. The resulting GO solid was dried at $60^{\circ} \mathrm{C}$ overnight.

\subsubsection{Synthesis of $\mathrm{Pd}$ nanoparticles stabilized} by polyvinylpyrrolidone (Pd/PVP)

In typical experimental procedure, catalysts with different palladium loadings $(5,10,20$ wt\%) on polyvinylpyrrolidone (PVP) were prepared. $30 \mathrm{~mL}$ mixture of deionized water and ethylene glycol (1:1) and the relevant weight of polyvinylpyrrolidone (PVP) based on a total weight of $100 \mathrm{mg}$ of catalyst were sonicated for 1.5 h. Then, different volumes (97, 194, 388,
$582 \mu \mathrm{L}$, respectively) of palladium nitrate solution were added to the sonicated solution and stirring was maintained for $1.5 \mathrm{~h}$. Then, the solution was heated using microwave for $30 \mathrm{~s}$, and then the catalyst is dried.

\subsubsection{Synthesis of Pd nanoparticles}

Palladium nitrate $(194 \mu \mathrm{L}$ was added to 50 $\mathrm{mL}$ mixture of deionized water and ethylene glycol (1:1), and the solution was then sonicated for $1 \mathrm{~h}$ and stirred for another $1 \mathrm{~h}$. The entire mixture was heated via a microwave oven for $30 \mathrm{~s}$, followed by filtering and washing with deionized water and finally ethanol, and then dried.

2.1.4 Synthesis of Pd/PVP nanoparticles supported on reduced Graphene Oxide nanosheets (Pd-PVP/rGO)

An amount of $60 \mathrm{mg}$ of graphite oxide was added to a solution of PVP in $50 \mathrm{~mL}$ deionized water and sonicated at room temperature for 1 h. After the sonication time was completed, Palladium nitrate (10 wt.\% in 10 wt.\% $\mathrm{HNO}_{3}$, $99.999 \%, 1000 \mu \mathrm{L})$ was added to the resulting mixture and the entire solution was stirred for 3 h. Ethylene glycol was slowly added as a stabilizer and a reducing agent to this mixture at ambient temperature and the solution was further heated under microwave irradiation (1000 W) for $120 \mathrm{~s}$. The final dark black color solution was filtered and the resulting black solid was washed with hot deionized water $(40 \mathrm{~mL}) 5$ times, ethanol $(10 \mathrm{~mL} 5$ times, and then dried overnight in the oven at $80^{\circ} \mathrm{C}$.

Table 1. Conversion percentage for different ratios of $\mathrm{Pd} / \mathrm{PVP}$ and $\mathrm{Pd}-\mathrm{PVP} / \mathrm{rGO}$

\begin{tabular}{|c|c|c|c|c|c|c|}
\hline Catalysts & $\begin{array}{c}\text { Temp. } \\
\left({ }^{\circ} \mathrm{C}\right)\end{array}$ & $\begin{array}{l}\text { Time } \\
\text { (min.) }\end{array}$ & $\begin{array}{c}\text { Conversion } \\
(\%)^{a^{*}}\end{array}$ & $\begin{array}{c}\text { Conversion } \\
(\%)^{b^{*}}\end{array}$ & $\begin{array}{c}\text { Conversion } \\
(\%)^{c^{*}}\end{array}$ & $\begin{array}{c}\text { Conversion } \\
(\%)^{d^{*}}\end{array}$ \\
\hline \multirow{3}{*}{$5 \mathrm{wt} \% \mathrm{Pd}-\mathrm{PVP}$} & 80 & 12 & 30 & 60 & 42 & 55 \\
\hline & 100 & 12 & 64 & 78 & 68 & 75 \\
\hline & 120 & 12 & 80 & 90 & 84 & 85 \\
\hline \multirow{3}{*}{$10 \mathrm{wt} \% \mathrm{Pd} / \mathrm{PVP}$} & 80 & 12 & 52 & 65 & 62 & 60 \\
\hline & 100 & 12 & 72 & 88 & 82 & 84 \\
\hline & 120 & 12 & 88 & 98 & 92 & 88 \\
\hline \multirow{3}{*}{$20 \mathrm{wt} \% \mathrm{Pd} / \mathrm{PVP}$} & 80 & 12 & 62 & 75 & 68 & 70 \\
\hline & 100 & 12 & 82 & 96 & 88 & 85 \\
\hline & 120 & 12 & 90 & 94 & 96 & 100 \\
\hline Pd nanoparticles & 120 & 12 & $<40$ & $<40$ & $<40$ & $<40$ \\
\hline
\end{tabular}

a,b Conversion was calculated for Heck and Suzuki cross coupling reactions using ( 1 mol\%) Pd/PVP catalyst, respectively.

c,d Conversion was calculated for Heck and Suzuki cross coupling reactions using (1 mol\%) Pd-PVP/rGO catalyst, respectively.

* Conversions were determined by GC-MS. 


\subsection{Catalyst Characterization}

Transmission electron microscopy (TEM) images were taken using A JEOL JEM-1230 electron microscope. Thermo Fisher Scientific ESCALAB was used to perform the X-ray photoelectron spectroscopy (XPS) analysis.

\subsection{General Procedure for Suzuki and Heck Cross-Coupling Reactions}

Aryl halide (0.32 mmol, 1 eq.) was dissolved in a mixture containing $4 \mathrm{~mL}$ deionized water and ethanol (1:1) as a solvent system. Both aryl boronic acid (0.382 mmol, 1.2 eq.) and potassium carbonate $(0.96 \mathrm{mmol}, 3 \mathrm{eq}$.) were also added to the deionized water-ethanol mixture. Pd$\mathrm{PVP} / \mathrm{rGO}(1 \mathrm{~mol} \%)$ catalyst was added and the mixture was heating via microwave at $120{ }^{\circ} \mathrm{C}$ for $12 \mathrm{~min}$. Finally, the resulting mixture was then extracted with $\mathrm{CH}_{2} \mathrm{Cl}_{2}$ and the solid product was dried.

\subsection{General Procedure for Catalyst Recycling}

Aryl halide (0.32 mmol, 1 eq.) was dissolved in a $6 \mathrm{~mL}$ mixture of deionized water and ethanol (1:1). Both aryl boronic acid $(0.382 \mathrm{mmol}$,
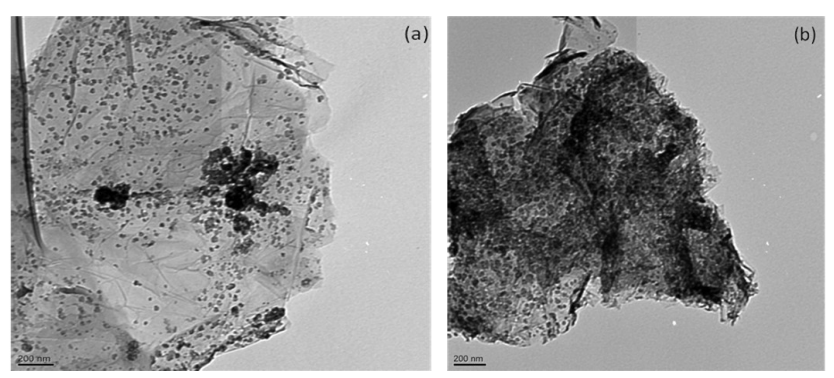

Figure 1. TEM-images of Pd-PVP/rGO (a) Before the reaction, and (b) After $5^{\text {th }}$ run of the reaction.
1.2 eq.) and potassium carbonate $(0.96 \mathrm{mmol}, 3$ eq.) were also added to the deionized waterethanol mixture. Pd-PVP/rGO (1 mol \%) catalyst was added and the mixture. Then, the mixture is heated at the assigned temperature and time as presented in Table 1. After the reaction was completed, the catalyst was completely removed and then used again.

\section{Results and Discussion}

Suzuki cross-coupling reaction of bromobenzene and phenyl boronic acid in 50 vol. \% aqueous ethanol under various reaction conditions was investigated using microwave reactor (Scheme 1). The temperature impact on the catalytic activity was studied through implementing the reaction at constant reaction time (12 min) and different temperatures $(80,100$, $120{ }^{\circ} \mathrm{C}$ ) using the synthesized Pd-PVP/rGO catalysts (1 mol\%) and compared to using Pd/PVP catalyst $(1 \mathrm{~mol} \%)$ in absence of the reduced graphene oxide.

It was confirmed experimentally that increasing temperature lead to higher conversion to the product and the maximum conversion was found to be obtained at $120^{\circ} \mathrm{C}$. This profile was similar for $\mathrm{Pd} / \mathrm{PVP}$ and $\mathrm{Pd}-\mathrm{PVP} / \mathrm{rGO}$ catalysts with different $\mathrm{Pd}$ loading percents on the solid support (5, 10, and $20 \mathrm{wt} \%)$ as shown in Table 1. It was found that increasing palladium loading $(5,10$, and $20 \mathrm{wt} \%$ ) improves the conversion as the maximum product conversion was found to be $100 \%$ for $20 \mathrm{wt} \%$ Pd loading at $120{ }^{\circ} \mathrm{C}$. It was also found experimentally that further increase in palladium loading (30 $\mathrm{wt} \%$ and higher) has a negative influence on the catalytic performance as the conversion was less than $60 \%$ and this may be due to the harmful influence of the agglomeration that

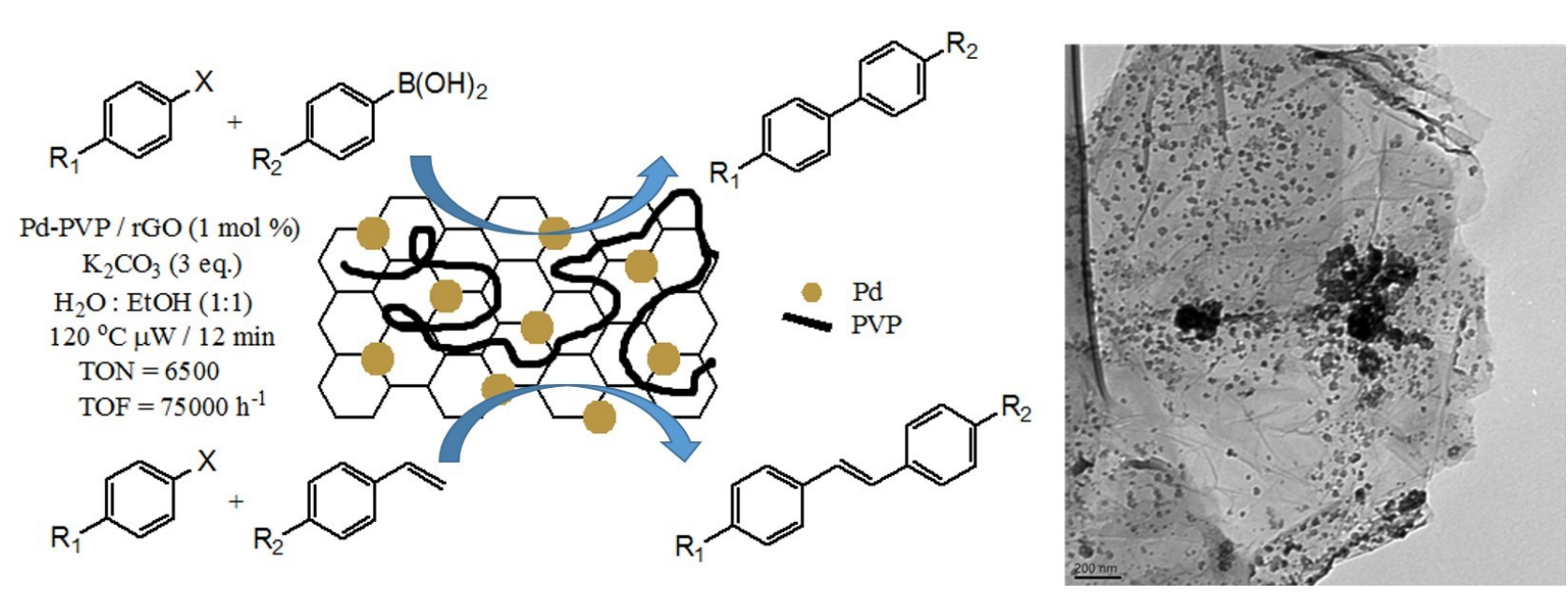

Scheme 1. Graphical abstract of using Pd-PVP/rGO in cross-coupling reaction 


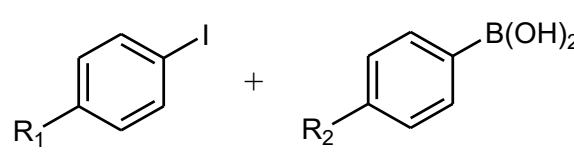

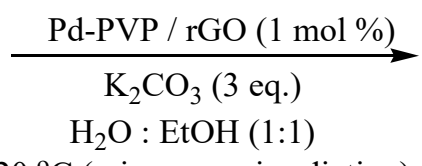

$\mathrm{H}_{2} \mathrm{O}:$ EtOH (1:1)

$12 \mathrm{~min}$<smiles>[R]c1ccc(-c2ccc([R])cc2)cc1</smiles>

Scheme 2. Suzuki cross coupling reactions using Pd-PVP/rGO catalyst.<smiles>Ic1ccccc1</smiles><smiles>C=Cc1ccccc1</smiles>

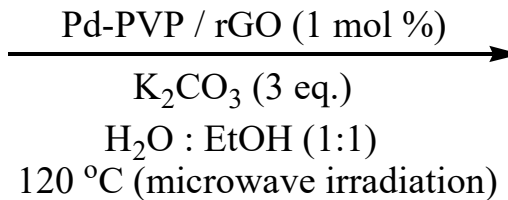
$12 \mathrm{~min}$<smiles>Ic1ccccc1</smiles><smiles>C#Cc1ccccc1</smiles>

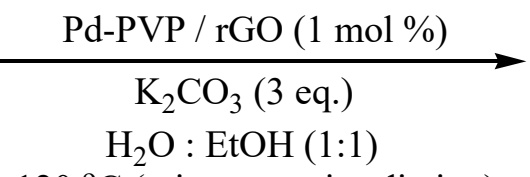
$120{ }^{\circ} \mathrm{C}$ (microwave irradiation) $12 \mathrm{~min}$<smiles>C(=C/c1ccccc1)\c1ccccc1</smiles>

$100 \%$<smiles>C(#Cc1ccccc1)c1ccccc1</smiles>

$100 \%$

Scheme 3. Reactivity of Pd-PVP/rGO catalyst towards Heck and Sonogashira coupling reactions

Table 2. Suzuki cross coupling reactions with various substrates using Pd/PVP and Pd-PVP/rGO catalyst

Entry

*Aryl halide (0.32 mmol, 1 eq.), aryl boronic acid (0.382 mmol, 1.2 eq.), potassium carbonate (0.96 mmol, 3 eq.), and catalyst (1 $\mathrm{mol} \%)$ in $4 \mathrm{~mL}\left(\mathrm{H}_{2} \mathrm{O}\right.$ : EtOH) (1:1) were heated at $120^{\circ} \mathrm{C}$ (microwave irradiation) for $12 \mathrm{~min}$. Conversions were determined by GC-MS.

a Conversion was calculated for Suzuki cross coupling reactions using ( $1 \mathrm{~mol} \%)$ Pd/PVP catalyst.

${ }^{\mathrm{b}}$ Conversion was calculated for Suzuki cross coupling reactions using (1 mol\%) Pd-PVP/rGO catalyst. 
happened due to the increase of palladium loading on the support surface.

It is remarkable to note that performing the reaction under the optimum conditions but with using $1 \mathrm{~mol} \% \mathrm{Pd}$ nanoparticles instead of $1 \mathrm{~mol} \% \mathrm{Pd}-\mathrm{PVP} / \mathrm{rGO}$ lead to a lower conversion (less than $40 \%$ ) and this confirms the decisive role played by in preventing the agglomeration of palladium nanoparticles and hence minimize the decrease in the catalyst activity [60-67]. The diversity of several substrates using $\mathrm{Pd} / \mathrm{PVP}$ and Pd-PVP/rGO catalysts has been explored in Tables 2 and 3. However, the diversity study of substrates in Heck cross-coupling reaction using both $\mathrm{Pd} / \mathrm{PVP}$ and $\mathrm{Pd}-\mathrm{PVP} / \mathrm{rGO}$ catalysts is explored in Table 4. It is obvious from the obtained data that using reduced Gra- phene oxide (rGO) as a support has a great influence on the catalytic activity due to the high surface area of Graphene $\left(2630 \mathrm{~m}^{2} / \mathrm{g}\right)$.

From the study of TEM images, the effect of using graphene enabled us to obtain well dispersed and smaller nanoparticles of size (10 $\pm 2 \mathrm{~nm}$ ) as in Figure 1-a. The TEM images here is completely matching with the high catalytic activity obtained from the experimental results in case of $\mathrm{Pd} / \mathrm{PVP}$ and $\mathrm{Pd}-\mathrm{PVP} / \mathrm{rGO}$ when compared with the same catalysts after the fifth run which is due to the agglomeration process of the particles that probably took place after reaction as shown in Figure 1-b. Figure 2 displays XRD pattern of Pd-PVP supported on reduced graphene oxide (rGO) that was prepared by microwave assisted synthesis. There is an

Table 3. Diversity of Suzuki cross coupling reactions using Pd/PVP and Pd-PVP/rGO catalyst<smiles></smiles>

*Aryl halide (0.32 mmol), aryl boronic acid ( $0.382 \mathrm{mmol}, 1.2$ eq.), potassium carbonate (0.96 mmol, 3 eq.), and catalyst (1 mol $\%)$ in $4 \mathrm{~mL}\left(\mathrm{H}_{2} \mathrm{O}: \mathrm{EtOH}\right)(1: 1)$ were heated at $120{ }^{\circ} \mathrm{C}$ (microwave irradiation) for $12 \mathrm{~min}$.

Conversions were determined by GC-MS.

a Conversion was calculated for Suzuki cross coupling reactions using (1 mol\%) Pd/PVP catalyst.

b Conversion was calculated for Suzuki cross coupling reactions using ( $1 \mathrm{~mol} \%)$ Pd-PVP/rGO catalyst. 
Table 4. Diversity of Heck coupling reactions using $\mathrm{Pd} / \mathrm{PVP}$ and $\mathrm{Pd}-\mathrm{PVP} / \mathrm{rGO}$ catalyst<smiles>[R]c1ccc(C=C)cc1</smiles>
$120{ }^{\circ} \mathrm{C}$ (microwave irradiation) $12 \mathrm{~min}$

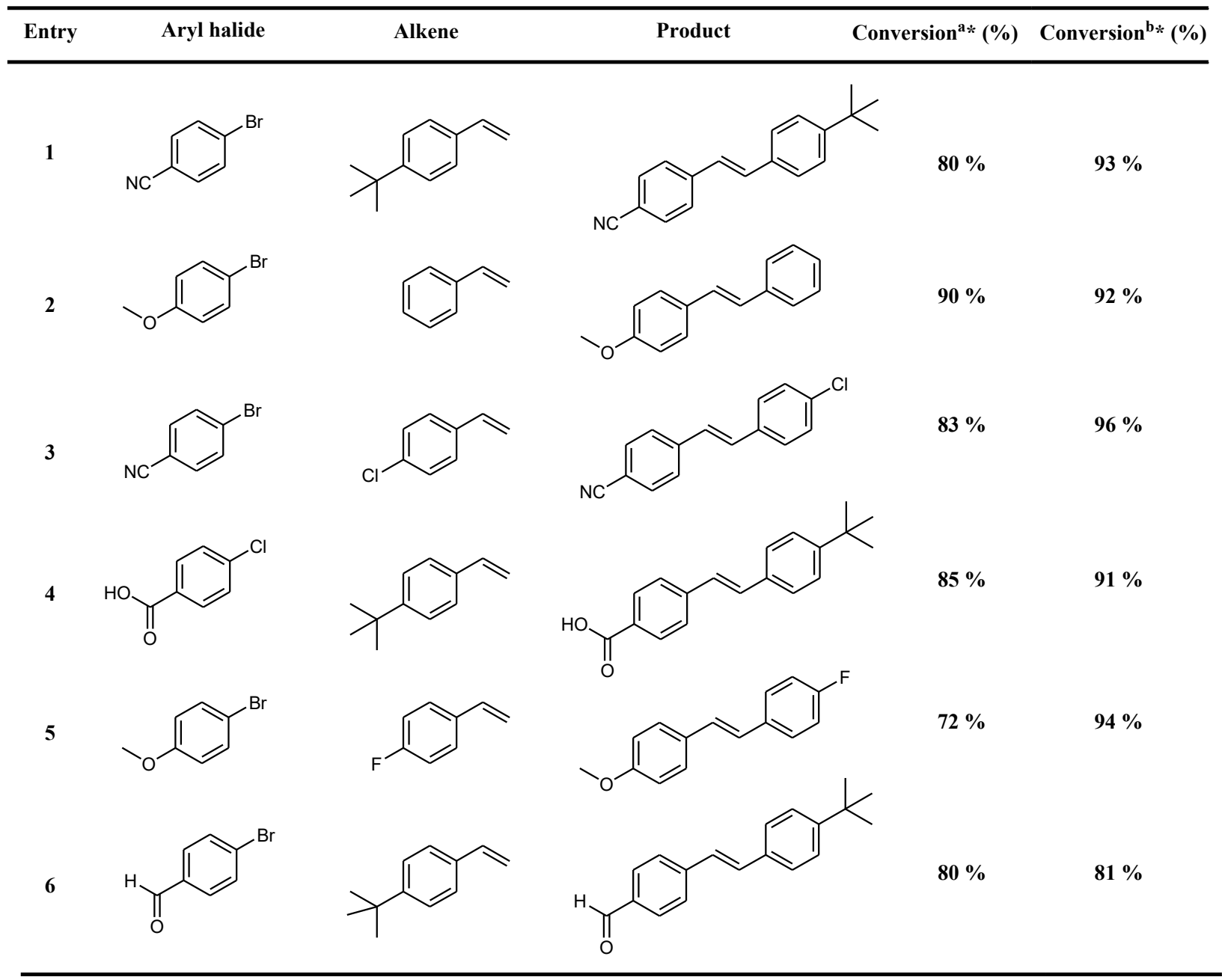

* Aryl bromide $(0.32 \mathrm{mmol})$, alkene $(0.64 \mathrm{mmol})$, potassium carbonate $(0.96 \mathrm{mmol})$, and catalyst $(1 \mathrm{~mol} \%)$ in $4 \mathrm{~mL}\left(\mathrm{H}_{2} \mathrm{O}\right.$ : EtOH) (1:1) were heated at $120{ }^{\circ} \mathrm{C}$ (microwave irradiation) for $12 \mathrm{~min}$. Conversions were determined by GC-MS.

a Conversion was calculated for Heck cross coupling reactions using (1 mol\%) Pd/PVP catalyst.

b Conversion was calculated for Heck cross coupling reactions using (1 mol\%) Pd-PVP/rGO catalyst. 
additional peak at 26.1 (002) ascribed to reduced graphene oxide (rGO). This is an indication of reduction of graphene oxide (GO) into reduced graphene oxide (rGO). Palladium content in Pd-PVP/rGO catalysts was determined via using inductively coupled plasma (ICPOES) and it was confirmed to be $3.5,6.5,14$ wt\% Pd compared to prepared $5 \mathrm{wt} \%, 10 \mathrm{wt} \%$, and $20 \mathrm{wt} \%$ of $\mathrm{Pd} / \mathrm{PVP} / \mathrm{rGO}$, respectively. XRD pattern of Pd-PVP/rGO catalyst shows a sharp diffraction peak at $2 \theta$ of $40^{\circ}$ which is characteristic to palladium as in Figure 2.

XPS technique is widely used as a more reliable and sensitive approach for implementing the surface analysis of oxides than XRD [63]. The surface composition of the prepared catalysts was characterized using XPS as in Figure 3 for the $20 \mathrm{wt} \% \mathrm{Pd}-\mathrm{PVP} / \mathrm{rGO}$ catalyst. All the samples due to carbon contamination have $\mathrm{C} 1 \mathrm{~s}$ binding energy around $284.5 \mathrm{eV}$. As shown in

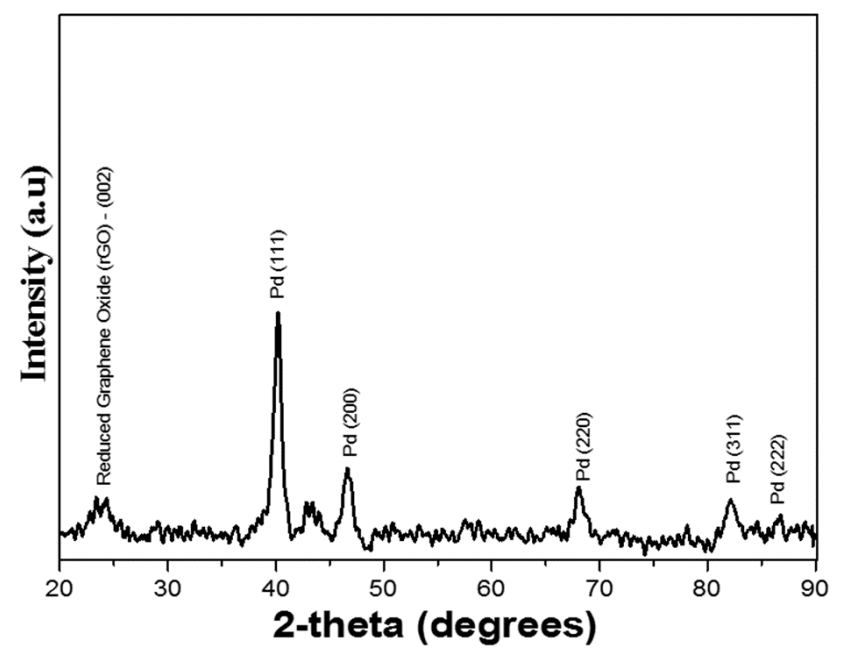

Figure 2. XRD pattern of Pd-PVP/rGO nanoparticles

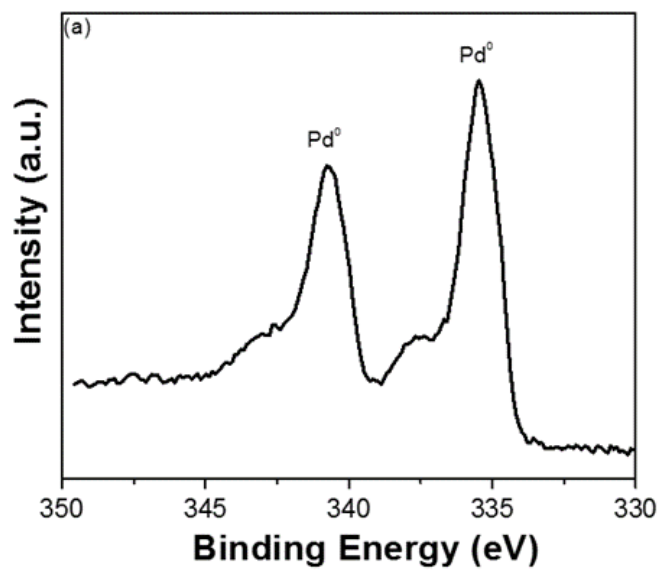

Figure 3a, the binding energy of $\mathrm{Pd} 3 \mathrm{~d}^{5 / 2}$ was $334.8 \mathrm{eV}$, and $\mathrm{Pd} 3 \mathrm{~d}^{3 / 2}$ was $340.1 \mathrm{eV}$ indicating that the Pd was ascribed to Pd ${ }^{0}$. Similarly, the binding energy of $\mathrm{Pd} 3 \mathrm{~d}^{3 / 2}$ was $341.38 \mathrm{eV}$, and $\mathrm{Pd} 3 \mathrm{~d}^{5 / 2}$ was $336.23 \mathrm{eV}$ indicating that the $\mathrm{Pd}$ was present as $\mathrm{PdO}\left(\mathrm{Pd}^{2+}\right)$ which is in agreement with the data found in literature.

The catalyst recycling process was previously discussed in section 2.4 and is summarized in Table 5. Table 5 and Figure 4, confirm the high catalytic activity of the catalyst. The catalyst with $0.5 \mathrm{~mol} \%$ could be recycled up to four times with almost $100 \%$ conversion. On the other hand, increasing the mole percentage to $1 \mathrm{~mol} \%$, lead to recycling for five times with conversion nearly $100 \%$ with excellent turnover number (6500) and turnover frequency of $78000 \mathrm{~h}^{-1}$. The deactivation of $\mathrm{Pd}-\mathrm{PVP} / \mathrm{rGO}$ catalysts can be confirmed through TEM images of the catalyst after $5^{\text {th }}$ run. This is mainly due to the harmful effect of the agglomeration process of Pd nanoparticles on the support surface as in Figure 1-b. The extent of Pd leaching from prepared catalyst was investigated through performing the reaction in the presence of $0.5 \mathrm{~mol} \%$ catalyst at $120^{\circ} \mathrm{C}$ for $12 \mathrm{~min}$ using microwave assisted synthesis technique. After ( $5^{\text {th }}$ run), the resulting mixture was filtered and then the content of $\mathrm{Pd}$ was measured in the filtrate and it was found to be $185 \mathrm{ppm}$ based on the ICP-OES analysis.

\section{Conclusions}

The microwave-assisted synthesis approach could simply provide a facile and effective route with shorter reaction times than those reported by other methods. It is also worth to note that even very low loading of palladium were sufficient to catalyze the reactions however, better conversion was obtained while increasing the

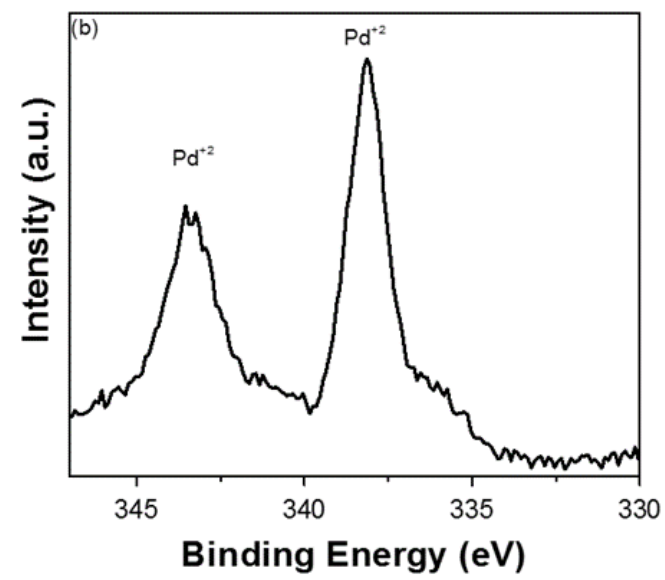

Figure 3. (a) XPS - Pd 3d before reaction, (b) XPS - Pd 3d after reaction for Pd-PVP/rGO 
loading. The mixture containing palladium nitrate, PVP, and graphite oxide sheets was reduced using ethylene glycol, which is used as a solvent and a reducing agent as well. It was confirmed that prepared catalysts were stable, recyclable, and highly active towards Suzuki, Heck, and Sonogashira cross - coupling reactions with nearly $100 \%$ conversion for the first run of the catalyst and with excellent turnover number (6500) and turnover frequency of 78000 $\mathrm{h}^{-1}$. Moreover, the catalysts are stable and can be reused up to five times with essentially no loss in the catalytic performance for a wide range of substrates under batch reaction conditions. The use of PVP offered an outstanding platform to design this efficient catalyst through forming strong interaction with graphene while maintaining excellent hydrophilic capability enabling not only ideal dispersion of rGO sheets in PVP matrix but also, excellent stabilization of the reduced Graphene oxide (rGO). From experimental data, it is well demonstrated that PVP has a significant effect as it is not only serve as a stabilizer but also as a mild reductant, that could contribute to the reduction of GO as well.

\section{Acknowledgements}

The authors are grateful to the support from The British University in Egypt (BUE) through Young Investigator Research Grant (YIRG) awarded to Dr. Hany A. Elazab. The authors also confirm that there is no conflict of interest.

Table 5. Recycling experiments for PdPVP/rGO catalyst using a concentration of $0.5,1 \mathrm{~mol} \%{ }^{\text {a }}$

\begin{tabular}{ccc}
\hline Run & $\begin{array}{c}\text { Conversion } \\
(\%)^{\mathrm{a}, \mathrm{b}} \\
(0.5 \mathrm{~mol} \%)\end{array}$ & $\begin{array}{c}\text { Conversion } \\
(\%)^{\mathrm{a}, \mathrm{b}} \\
(1 \mathrm{~mol} \%)\end{array}$ \\
\hline 1 & 100 & 100 \\
2 & 98 & 100 \\
3 & 95 & 98 \\
4 & 93 & 95 \\
5 & 92 & 94 \\
6 & 84 & 88 \\
\hline
\end{tabular}

a Bromobenzene $(50 \mathrm{mg}, 0.32 \mathrm{mmol})$, boronic acid $(47$ $\mathrm{mg}, 0.382 \mathrm{mmol}, 1.2 \mathrm{eq}$.$) , potassium carbonate (133 \mathrm{mg}$, $0.96 \mathrm{mmol}, 3$ eq.), and Pd-PVP/rGO (0.5 mol\%) or (1 mol\%) in $4 \mathrm{~mL}\left(\mathrm{H}_{2} \mathrm{O}\right.$ : EtOH) $(1: 1)$ were heated at $120{ }^{\circ} \mathrm{C}$ (microwave irradiation) for $12 \mathrm{~min}$.

b Conversions were determined by GC-MS.

\section{References}

[1] Chen, S., Cheng, B., Ding, C. (2006). Synthesis and Characterization of Poly(vinyl pyrrolidone)/Reduced Graphene Oxide Nanocomposite. Journal of Macromolecular Science, Part B, 54(4): 481-491.

[2] De Souza, A.L.F. (2008). Microwave- and ultrasound-assisted Suzuki Miyaura crosscoupling reactions catalyzed by $\mathrm{Pd} / \mathrm{PVP}$. Tetrahedron Letters, 49(24): 3895-3898.

[3] Durap, F. (2009). New route to synthesis of PVP stabilized palladium(0) nanoclusters and their enhanced catalytic activity in Heck and Suzuki cross coupling reactions. Applied Organometallic Chemistry, 23(12): 498-503.

[4] Gniewek, A. (2005). Pd-PVP colloid as catalyst for Heck and carbonylation reactions: TEM and XPS studies. Journal of Catalysis, 229(2): 332-343.

[5] Ananikov, V.P. (2007). New approach for sizeand shape-controlled preparation of pd nanoparticles with organic ligands. Synthesis and application in catalysis, Journal of the American Chemical Society, 129(23): 7252-7260.

[6] Ashfield, L. (2007). Reductive car-bonylation an efficient and practical catalytic route for the conversion of aryl halides to aldehydes. Organic Process Research \& Development, 11(1): 39-43.

[7] Guillen, E. (2009). Pd-activated carbon catalysts for hydrogenation and Suzuki reactions. Applied Catalysis A: General, 368(1-2): 113120.

[8] Karousis, N. (2008). Carbon nanotubes decorated with palladium nanoparticles: Synthesis, characterization, and catalytic activity. Journal of Physical Chemistry C, 112(35): 13463-13469.

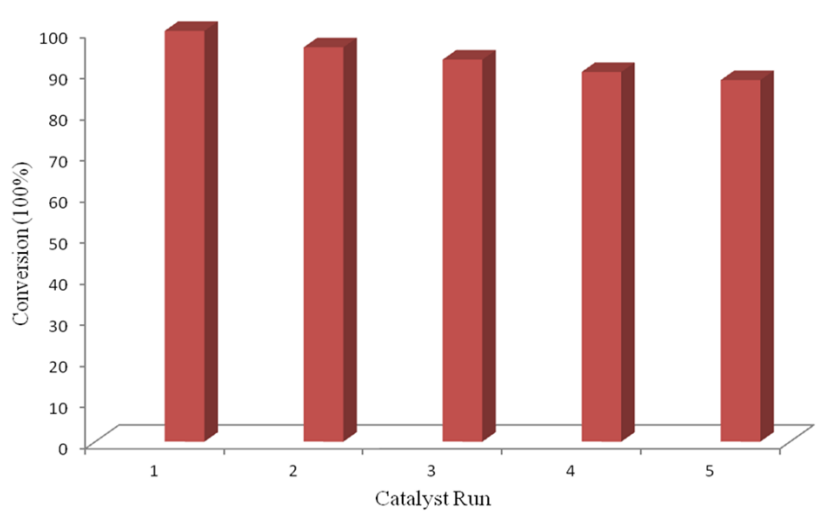

Figure 4. Recycling experiments of $\mathrm{Pd}$ PVP/rGO catalyst for Suzuki cross-coupling reaction 
[9] Leonhardt, S.E.S. (2006). Chitosan as a support for heterogeneous Pd catalysts in liquid phase catalysis. Applied Catalysis A: General, 379(1-2): 30-37.

[10] Li, Y. (2006). Palladium nanoparticlegraphene hybrids as active catalysts for the Suzuki reaction. Nano Research, 3(6): 429437.

[11] Elazab, H. (2014). Microwave-assisted synthesis of $\mathrm{Pd}$ nanoparticles supported on $\mathrm{FeO}$, $\mathrm{CoO}$, and $\mathrm{Ni}(\mathrm{OH})$ nanoplates and catalysis application for CO oxidation. Journal of $\mathrm{Na}$ noparticle Research, 16(7): 1-11.

[12] Elazab, H. (2017). The Effect of Graphene on Catalytic Performance of Palladium Nanoparticles Decorated with $\mathrm{FeO}, \mathrm{CoO}$, and $\mathrm{Ni}(\mathrm{OH})$ : Potential Efficient Catalysts used for Suzuki Cross-Coupling. Catalysis Letters, 147(6): 1510-1522.

[13] Elazab, H.A. (2017). The continuous synthesis of $\mathrm{Pd}$ supported on $\mathrm{Fe}_{3} \mathrm{O}_{4}$ nanoparticles: A highly effective and magnetic catalyst for $\mathrm{CO}$ oxidation. Green Processing and Synthesis, 6(4): 413-424.

[14] Elazab, H.A., Sadek, M.A., El-Idreesy, T.T. (2018). Microwave-assisted synthesis of palladium nanoparticles supported on copper oxide in aqueous medium as an efficient catalyst for Suzuki cross-coupling reaction, Adsorption Science \& Technology, 36(5-6): 1352-1365.

[15] Elazab, H.A. (2015). Highly efficient and magnetically recyclable graphene-supported $\mathrm{Pd} / \mathrm{Fe}_{3} \mathrm{O}_{4}$ nanoparticle catalysts for Suzuki and Heck cross-coupling reactions. Applied Catalysis A: General, 491: 58-69.

[16] Mohsen, W., Sadek, M.A., Elazab, H. (2017). Green synthesis of copper oxide nanoparticles in aqueous medium as a potential efficient catalyst for catalysis applications. International Journal of Applied Engineering Research, 12(24): 14927-14930.

[17] Bondioli, F. (2008). Synthesis of Zirconia Nanoparticles in a Continuous-Flow Microwave Reactor. Journal of the American Ceramic Society, 91(11): 3746-3748.

[18] Fukui, K. (2012). Mechanism of synthesis of metallic oxide powder from aqueous metallic nitrate solution by microwave denitration method. Chemical Engineering Journal, 211: 1-8.

[19] Glasnov, T.N., Findenig, S., Kappe, C.O. (2009). Heterogeneous versus Homogeneous Palladium Catalysts for Ligandless MizorokiHeck Reactions: A Comparison of Batch/Microwave and Continuous-Flow Processing. Chemistry - A European Journal, 15(4): 1001-1010.
[20] Kirschning, A., Kupracz, L., Hartwig, J. (2012). New Synthetic Opportunities in Miniaturized Flow Reactors with Inductive Heating. Chemistry Letters, 41(6): 562-570.

[21] Malewicz, M. (2009). Synthesis of Zinc Oxide Nanotiles by Wet Chemical Route Assisted by Microwave Heating. Electronics Technology, 15(3): 47-50.

[22] Pourmortazavi, S.M. (2012). Synthesis, structure characterization and catalytic activity of nickel tungstate nanoparticles. Applied Surface Science, 263: 745-752.

[23] Elazab, H.A., Sadek, M.A., El-Idreesy, T.T. (2018). Microwave-assisted synthesis of palladium nanoparticles supported on copper oxide in aqueous medium as an efficient catalyst for Suzuki cross-coupling reaction. Adsorption Science \& Technology, 36(5-6): 1352 1365 .

[24] Yu, X.H. (2006). Research Progress of Nanostructured Materials for Heterogeneous Catalysis. Current Nanoscience, 7(4): 576586.

[25] Horikoshi, S. (2006). On the Generation of Hot-Spots by Microwave Electric and Magnetic Fields and Their Impact on a Microwave-Assisted Heterogeneous Reaction in the Presence of Metallic Pd Nanoparticles on an Activated Carbon Support. Journal of Physical Chemistry C, 115(46): 23030-23035.

[26] Falcon, H. (2010). Large-scale synthesis of porous magnetic composites for catalytic applications, in Scientific Bases for the Preparation of Heterogeneous Catalysts: Proceedings of the $10^{\text {th }}$ International Symposium, E.M. Gaigneaux, 347-350.

[27] Chen, S.T. (2012). Synthesis of Pd/Fe3O4 Hybrid Nanocatalysts with Controllable Interface and Enhanced Catalytic Activities for CO Oxidation. Journal of Physical Chemistry C, 116(23): 12969-12976.

[28] Moussa, S., Abdelsayed, V., El-Shall, M.S. (2011). Laser synthesis of Pt, Pd, CoO and $\mathrm{Pd}-\mathrm{CoO}$ nanoparticle catalysts supported on graphene. Chemical Physics Letters, 510(4-6): 179-184.

[29] Qiu, G.H. (2011). Microwave-Assisted Hydrothermal Synthesis of Nanosized alpha-Fe2O3 for Catalysts and Adsorbents. Journal of Physical Chemistry C, 115(40): 19626-19631.

[30] Wang, H.L. (2010). Ni(OH) 2 Nanoplates Grown on Graphene as Advanced Electrochemical Pseudocapacitor Materials. Journal of the American Chemical Society, 132(21): 7472-7477. 
[31] Wang, H.L. (2010). Nanocrystal Growth on Graphene with Various Degrees of Oxidation. Journal of the American Chemical Society, 132(10): 270-285.

[32] Kalbasi, R.J., Negahdari, M. (2006). Synthesis and characterization of mesoporous poly(N-vinyl-2-pyrrolidone) containing palladium nanoparticles as a novel heterogeneous organocatalyst for Heck reaction. Journal of Molecular Structure, 1063: 259-268.

[33] Martins, D.d.L. (2009). Heck reactions catalyzed by $\operatorname{Pd}(0)$-PVP nanoparticles under conventional and microwave heating. Applied Catalysis A: General, 408(1): 47-53.

[34] Sheng, L. (2006). PVP-coated graphene oxide for selective determination of ochratoxin A via quenching fluorescence of free aptamer. Biosensors and Bioelectronics, 26(8): 3494-3499.

[35] Zhang, J. (2010). Microwave-assisted synthesis of $\mathrm{Pd}$ nanoparticles and their catalysis application for Suzuki cross-coupling re-actions. Inorganic and Nano-Metal Chemistry, 47(5): 672-676.

[36] Zhang, X. (2006). Polyvinyl pyrrolidone modified graphene oxide for improving the mechanical, thermal conductivity and solvent resistance properties of natural rubber. $R S C$ Advances, 6(60): 54668-54678.

[37] Zhang, Y. (2011). One-step synthesis of Polyvinylpyrrolidone-reduced graphene oxide- $\mathrm{Pd}$ nanoparticles for electrochemical sensing. Journal of Materials Science, 51(13): $6497-$ 6508.

[38] Nicolaou, K.C., Bulger, P.G., Sarlah, D. (2005). Palladium-catalyzed cross-coupling reactions in total synthesis. Angewandte Chemie-International Edition, 44(29): 4442 4489.

[39] Ashraf, B., Elazab, H. (2018). Preparation and characterization of decorative and heat insulating floor tiles for buildings roofs. International Journal of Engineering and Technology (UAE), 7(3): 1295-1298.

[40] Elazab H.A. (2018). Laser vaporization and controlled condensation (LVCC) of graphene supported $\mathrm{Pd} / \mathrm{Fe}_{3} \mathrm{O}_{4}$ nanoparticles as an efficient magnetic catalysts for Suzuki Cross Coupling. Biointerface Research in Applied Chemistry, 8(3): 3314-3318.

[41] Elazab, H.A. (2018). The catalytic activity of copper oxide nanoparticles towards carbon monoxide oxidation catalysis: microwave assisted synthesis approach, Biointerface Research in Applied Chemistry, 8(3): p. 32783281.

[42] Elazab, H.A., Radwan, M.A., El-Idreesy, T.T. (2018). Facile Microwave-Assisted Synthetic Approach to Palladium Nanoparticles Sup- ported on Copper Oxide as an Efficient Catalyst for Heck and Sonogashira CrossCoupling Reactions, International Journal of Nanoscience, 17(3): 1850032-1850040.

[43] Ceylan, S. (2011). Inductive Heating with Magnetic Materials inside Flow Reactors. Chemistry - A European Journal, 17(6): 18841893.

[44] Gupta, A. (2011). Synthesis and Ink-Jet Printing of Highly Luminescing Silicon Nanoparticles for Printable Electronics. Journal of Nanoscience and Nanotechnology, 11(6): 5028-5033.

[45] Nishioka, M. (2011). Continuous synthesis of monodispersed silver nanoparticles using a homogeneous heating microwave reactor system. Nanoscale, 3(6): 2621-2626.

[46] Shviro, M., Zitoun, D. (2013). Nickel nanocrystals: fast synthesis of cubes, pyramids and tetrapods. RSC Advances, 3(5): 13801387.

[47] Beckert, M. (2015). Nitrogenated graphene and carbon nanomaterials by carbonization of polyfurfuryl alcohol in the presence of urea and dicyandiamide. Green Chemistry, 17(2): 1032-1037.

[48] Kumar, S. (2015). Graphene, carbon nanotubes, zinc oxide and gold as elite nanomaterials for fabrication of biosensors for healthcare. Biosensors \& Bioelectronics, 70: 498-503.

[49] Neri, G. (2015). Engineering of carbon based nanomaterials by ring-opening reactions of a reactive azlactone graphene platform. Chemical Communications, 51(23): 4846-4849.

[50] Mankarious, R.A., Elazab, H. (2017). Bulletproof vests/shields prepared from composite material based on strong polyamide fibers and epoxy resin. Journal of Engineering and Applied Sciences, 12(10): 2697-2701.

[51] Mostafa, A.R., Omar, H.A.-S., Elazab, H.A. (2017). Preparation of Hydrogel Based on Acryl Amide and Investigation of Different Factors Affecting Rate and Amount of Absorbed Water. Agricultural Sciences, 8(2): 1118.

[52] Radwan, M.A., Elazab, H.A. (2017). Mechanical characteristics for different composite materials based on commercial epoxy resins and different fillers, Journal of Engineering and Applied Sciences, 12(5): 1179-1185.

[53] Samir, N.S., Elazab, H.A. (2018). Preparation and characterization of bullet-proof vests based on polyamide fibers. International Journal of Engineering and Technology (UAE), 7(3): 1290-1294. 
[54] Tang, Y., Yang, Z. (2011). Trapping of metal atoms in the defects on graphene. Journal of Chemical Physics, 13(5): 22-32.

[55] Wang, Q. (2012). Adsorption of oxygencontaining functional groups on free and supported graphene using point contact. Physical Review B, 85(8), 85-96.

[56] Wu, S.X. (2012). Synthesis of $\mathrm{Fe}_{3} \mathrm{O}_{4}$ and Pt nanoparticles on reduced graphene oxide and their use as a recyclable catalyst. Nanoscale, 4(7): 2478-2483.

[57] Xi, P.X. (2012). Surfactant free RGO/Pd nanocomposites as highly active heterogeneous catalysts for the hydrolytic dehydrogenation of ammonia borane for chemical hydrogen storage. Nanoscale, 4(18): 5597-5601.

[58] Zhou, M. (2011). Adsorption of gas molecules on transition metal embedded graphene: a search for high-performance graphene-based catalysts and gas sensors. Nanotechnology, 22(38): 124-134.

[59] Botas, C. (2013). Graphene materials with different structures prepared from the same graphite by the Hummers and Brodie methods. Carbon, 65: 156-164.

[60] Hummers, W.S., Offeman, R.E. (1958). Preparation of Graphitic Oxide. Journal of the American Chemical Society, 80(6): 1339-1339.

[61] Rovnick, N. (2002). Scottish anti-lawyer group mounts political challenge, Lawyer, 16(37): 414.

[62] You, S. (2013). Effect of synthesis method on solvation and exfoliation of graphite oxide. Carbon, 52: 171-180.
[63] Elazab, H.A. (2019). Optimization of the Catalytic Performance of $\mathrm{Pd} / \mathrm{Fe}_{3} \mathrm{O}_{4}$ Nanoparticles Prepared via Microwave-assisted Synthesis for Pharmaceutical and Catalysis Applications, Biointerface Research in Applied Chemistry, 9(1): 3794-3799.

[64] Elazab, H.A. (2019). Investigation of Microwave-assisted Synthesis of Palladium Nanoparticles Supported on $\mathrm{Fe}_{3} \mathrm{O}_{4}$ as an Efficient Recyclable Magnetic Catalysts for Suzuki Cross - Coupling, The Canadian Journal of Chemical Engineering, 97(5): 225-234.

[65] Zakaria, F., Radwan, M.A., Sadek, M.A., Elazab, H.A. (2018). Insulating material based on shredded used tires and inexpensive polymers for different roofs. International Journal of Engineering and Technology (UAE), 7(4):1983-1988.

[66] Nasser, R., Radwan, M.A., Sadek, M.A., Elazab, H.A. (2018). Preparation of insulating material based on rice straw and inexpensive polymers for different roofs. International Journal of Engineering and Technology (UAE), 7(4): 1989-1994.

[67] Ghobashy, M., Gadallah, M., El-Idreesy, T.T., Sadek, T.T., Elazab, H.A. (2018). Kinetic Study of Hydrolysis of Ethyl Acetate using Caustic Soda. International Journal of Engineering and Technology (UAE), 7(4): 19951999.

[68] Radwan, M.A., Rashad, M.A., Sadek, M.A., Elazab, H.A. (2019). Synthesis, Characterization and Selected Application of ChitosanCoated Magnetic Iron Oxide nanoparticles. Journal of Chemical Technology and Metallurgy, 54(2): 303-310. 